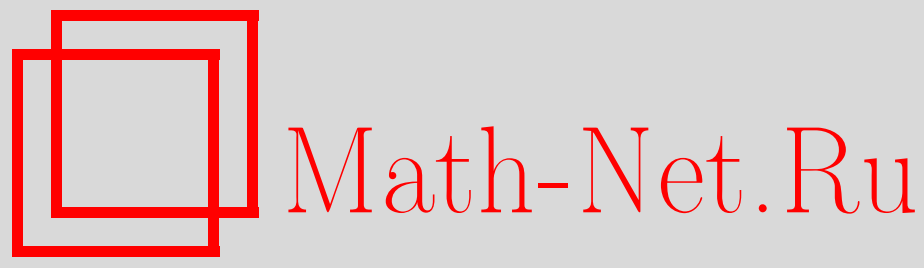

Т. А. Белкина, Ю. М. Кабанов, Э. Л. Пресман, О стохастической оптимальности для линейноквадратического регулятора, Теория вероятн. и ее примен., 2003, том 48, выпуск 4, 661-675

DOI: https://doi.org/10.4213/tvp250

Использование Общероссийского математического портала MathNet.Ru подразумевает, что вы прочитали и согласны с пользовательским соглашением

http: //www.mathnet.ru/rus/agreement

Параметры загрузки:

IP : 54.81 .137 .203

26 апреля 2023 г., 12:37:03

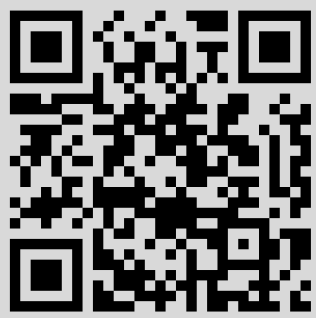


ТЕОРИЯ ВЕРОЯТНОСТЕЙ

(C) 2003 г. БЕЛКИНА Т. А., КАБАНОВ Ю. М.**, ПРЕСМАН Э. Л.*

\section{О СТОХАСТИЧЕСКОЙ ОПТИМАЛЬНОСТИ ДЛЯ ЛИНЕЙНО-КВАДРАТИЧЕСКОГО РЕГУЛЯТОРА ${ }^{1)}$}

В работе показывается, что оптимальное на бесконечном интервале времени марковское управление $\widehat{u}$ в стандартной задаче линейно-квадратического регулятора обладает следуюшим свойством. Существует такая константа $b_{*}$, что для любого $b>b_{*}$ процесс дефекта оптимального управления относительно любого конкурирующего управления, т.е. разность $J_{T}(\widehat{u})-J_{T}(u)$ между значением квадратического функционала $J_{T}(\widehat{u})$, отвечающим оптимальному на бесконечности управлению, и значением $J_{T}(u)$, отвечаюшим конкурируюшему управлению $u$, мажорируется на бесконечности функцией $b \ln T$, т.е. функция $b \ln T$ является верхней функцией для любого процесса дефекта. Этот результат в комбинации с примером линейно-квадратического регулятора, в котором при некотором $b>0$ функция $b \ln T$ не является верхней функцией для некоторого процесса дефекта, дает ответ на давно стоящий вопрос о наилучшей весовой функции для чувствительных вероятностных критериев. Приводимая постановка включает так называемую задачу оптимального «трекинга».

Ключевые слова и фразы: линейно-квадратический регулятор, оптимальность почти наверное, наблюдаемость, управляемость, уравнение Риккати, закон больших чисел для мартингалов, верхние функции, процесс Орнштейна-Уленбека.

\section{1. Введение}

Рассмотрим совокупность $\mathscr{Z}$ случайных процессов $z=\left(z_{t}\right)$, заданных на некотором стохастическом базисе. Пусть

$$
J_{T}(z):=F_{T}\left(z_{T}\right)+\int_{0}^{T} f_{t}\left(z_{t}\right) d t
$$

* ЦЭМИ РАН, Нахимовский пр., 47, 117. 418 Москва, Россия.

** Университет Безансона, Франция и ЦЭМИ РАН; Youri.Kabanov@descartes.univfcomte.fr

1) Работа выполнена при частичной поддержке Российского фонда фундаментальных исследований, грант 03-01-00479, РАН, грант 105 по 6-му конкурсу-экспертизе проектов молодых ученых, и НАTO, грант CRG-972115. 
где $f_{t}\left(z_{t}\right) \geqslant 0$ интерпретируется как текущие платежи, а $F_{T}\left(z_{T}\right)$ - как финальная плата. Следуя работе [9], мы говорим, что $\widehat{z}$ оптимально п.н. с весовой Функиией $g$ или, коротко, g-оптимально п.н., $g=\left(g_{t}\right)$ положительная невозрастающая функция, если для любого $z \in \mathscr{Z}$

$$
\limsup _{T \rightarrow \infty}\left(J_{T}(\widehat{z})-J_{T}(z)\right)^{+} g_{T}=0 .
$$

Эта постановка включает в себя многие частные случаи, встречающиеся в литературе. Обычно $\mathscr{Z}=\{(x, u): u \in \mathscr{U}\}$, где $x=x^{u}$ - решение стохастического уравнения, соответствующего управлению $u$, а $\mathscr{U}-$ множество допустимых управлений. Если весовая функция равна константе, то получается «overtaking» оптимальность п.н. (введенная в работе [15]). Если $g_{T}=1 / T$, то получается стохастическое долговременное среднее, которое изучалось для различных моделей (в том числе и с дискретным временем) многими авторами (см. [1], [3], [5], [11], [12], [15], [16], [18], [21], [23], а также книгу [6]). Другие близкие подходы можно найти в [14] («overtaking» оптимальность для математических ожиданий) и в [17], [2] (оптимальность по распределению с $g_{T}=T^{-1 / 2}$ ).

Концепция стохастической оптимальности естественным образом возникает в классической задаче на бесконечном интервале времени с ожидаемыми средними за единицу времени расходами:

$$
\limsup _{T \rightarrow \infty} \frac{1}{T} \mathbf{E} J_{T}(z) \rightarrow \min .
$$

В важном случае стохастического уравнения с постоянными коэффициентами оптимальный процесс $\widehat{z}$ эргодичен. Поскольку эргодическое среднее сходится при $T \rightarrow \infty$ к ожидаемому среднему значению, то можно надеяться, что $\widehat{z}$ лучше, чем любой другой эргодический $z$, также и в стохастическом смысле. В упомянутых выше работах было показано, что в рассматриваемых моделях $\widehat{z}$ на самом деле «лучше», чем любой конкурирующий управляемый процесс (и не только эргодический) при условии, что сравнение производится в смысле (2) с $g_{T}=1 / T$.

В большинстве существующих работ кандидат $\widehat{z}$ известен. Он возникает из решения некоторой стандартной задачи (например, (3)), а поэтому вопрос сводится к изучению семейства $\mathscr{D}$ процессов дефекта $\Delta_{T}:=J_{T}(\widehat{z})-J_{T}(z)$ (точнее, его положительной части), $z \in \mathscr{Z}$.

Для этой цели удобно использовать следующее определение.

Возрастающая функция $h$ является верхней функиией для совокупности процессов дефекта, соответствующих процессу $\widehat{z}$, если для любого $z \in \mathscr{Z}$ существует конечный (п.н.) момент времени, после которого функция $h$ мажорирует (п.н.) соответствующий процесс дефекта $\Delta$. Цель состоит в нахождении верхней функции $\widehat{h}$ наименьшего роста.

Очевидно, что весовая функция $1 / T$ соответствует закону больших чисел, и, в самом деле, мартингальная версия этого закона являлась 
основным средством в предшествующих работах (его использование в задачах оптимального управления восходит, по-видимому, к работе [10]). Поиск наиболее медленно убывающей весовой функции $g$, для которой $\widehat{z}$ все еще оптимально п.н. (начатый в работе [9]), или поиск самой медленно растущей $h$ выявляет связь изучаемого вопроса с более деликатными вероятностными результатами такими, как закон повторного логарифма.

Мы концентрируем наши усилия на классической задаче линейноквадратического регулятора и показываем, что при соответствуюших предположениях сушествует такая константа $b$, что $b \ln T$ является верхней функцией для семейства $\mathscr{D}$. Данная статья завершает исследования задачи линейно-квадратического регулятора со стохастическим критерием оптимальности. Такие исследования для не зависящих от времени коэффициентов проводились в работе [15], где в качестве конкурирующих к оптимальному управлению рассматривались только управления, задаваемые в виде синтеза, затем в работе [21] был рассмотрен скалярный случай, но с произвольными управлениями, в работах [9] и [20] результат был обобщен на многомерный случай с последовательным улучшением до $g_{T}=1 / T^{1 / 2+\varepsilon}$ и $g_{T}=1 / T^{\varepsilon}, \varepsilon>0$, соответственно.

Для удобства читателя сначала мы сформулируем результат для стандартной модели с постоянными коэффициентами:

$$
\begin{gathered}
d x_{t}=\left(A x_{t}+B u_{t}\right) d t+G d w_{t}, \quad x_{0}=\xi \\
f_{t}\left(x_{t}, u_{t}\right)=\left\|C x_{t}\right\|^{2}+\left\|u_{t}\right\|^{2}, \quad F_{T}\left(x_{T}, u_{T}\right)=0
\end{gathered}
$$

где $w$ является $p$-мерным винеровским процессом, $\xi \in \mathbf{R}^{n}, \mathscr{U}$ состоит из адаптированных $m$-мерных процессов, для которых (4) имеет смысл, а матрица $C$ симметрична и неотрицательно определена.

Пусть $\widehat{z}=(\widehat{x}, \widehat{u})$ - соответственно фазовый процесс и процесс управления, отвечающие оптимальному синтезу в задаче с критерием (3).

Теорема 1. Предположим, что пара $(A, B)$ стабилизируема, a $(A, C)$ вьявляема. Тогда существует такая не зависяиая от $\xi$ константа $b$, что $h_{T}=b \ln T$ является верхней функиией для семейства прочессов дефекта, отвечающего всем допустимьм управлениям.

Отметим, что предположения теоремы выполняются в случае, когда пара $(A, B)$ управляема, а пара $(A, C)$ наблюдаема (условия, которые рассматривались в предыдущих исследованиях [15], [9], [20]).

Точное описание $\widehat{u}$ и обсуждение предположений приводится ниже в разделах 2 и 4 . Сформулированная выше теорема выводится из более обцей теоремы, которая является основным результатом работы и формулируется для модели, с зависящими от времени коэффициентами, Хотя формулировка носит несколько технический характер, она проливает свет на свойства, необходимые для того, чтобы функция $b \ln T$ была 
верхней функцией для совокупности процессов дефекта, отвечающей самому широкому классу конкурирующих управлений. Более того, неоднородная по времени постановка позволяет рассмотреть практически важный случай «трекинга» (см. [14], где рассматривались эргодические конкурирующие управления).

Идея доказательства совпадает с идеей [20]: для процесса дефекта находится мажоранта, которая является суммой квадратичной нормы процесса Орнштейна-Уленбека и непрерывного супермартингала со сносом, пропорциональным квадратичной характеристике его мартингальной части. Результат следует из этого представления, поскольку вторая компонента либо имеет предел, либо стремится к минус бесконечности, в то время как первая компонента мажорируется логарифмической функцией (см. дополнение).

Напомним, что в [19] было показано, что в одномерном случае с $A=0, B=\frac{1}{2}, C=G=1$ существуют $z^{*}$ и такая константа $b>0$, что

$$
\limsup _{T \rightarrow \infty} \frac{J_{T}(\widehat{z})-J_{T}\left(z^{*}\right)}{\ln T}>b
$$

Заметим также, что более узкий класс конкурирующих управлений, порождающий только эргодические фазовые процессы, обладает даже так называемым свойством «оvertaking» оптимальности п.н. (см. [15]). С другой стороны, если процессы $\widehat{z}$ и $z$ определяются по различным винеровским процессам, то самая медленно растушцая функция имеет порядок $O\left(T^{1 / 2}\right)$ (см. [7]).

Рассмотрим значение $b_{*}$, которое является инфимумом тех $b$, для которых выполняется утверждение теоремы. Как найти значение $b_{*}$ ? Рассуждения в [19] дают некоторые оценки, но точное значение не известно даже для рассмотренного простого примера. Является ли функция $b_{*} \ln T$ верхней функцией для совокупности процессов дефекта? Этот вопрос также остается открытым.

\section{2. Основной результат}

Рассматривается модель

$$
\begin{gathered}
d x_{t}=\left(A_{t} x_{t}+B_{t} u_{t}\right) d t+l_{t} d t+G_{t} d w_{t}, \quad x_{0}=\xi \\
f_{t}\left(x_{t}, u_{t}\right)=\left\|C_{t} x_{t}\right\|^{2}+\left\|u_{t}\right\|^{2}+2 m_{t}^{\prime} x_{t}, \quad F_{T}\left(x_{T}, u_{T}\right)=\left\|F_{T} x_{T}\right\|^{2}+2 n_{T}^{\prime} x_{T}
\end{gathered}
$$

где $A, B, C, F, G, l, m$ и $n$ - ограниченные (измеримые) функции времени $t$. Классический результат (см. [8], [4]), который следует также из 
приводимого ниже тождества (23), утверждает, что для любого конечного $T$ оптимальное управление в задаче

$$
\mathbf{E} J_{T}(z) \rightarrow \min
$$

единственно, имеет вид синтеза и может быть описано следующим образом.

Пусть $\Pi^{T}=\left(\Pi_{t}^{T}\right)_{t \leqslant T}$ - функция со значениями в множестве симметричных неотрицательно определенных матриц, абсолютно непрерывная и удовлетворяющая уравнению Риккати

$$
\dot{\Pi}_{t}+\Pi_{t} A_{t}+A_{t}^{\prime} \Pi_{t}-\Pi_{t} B_{t} B_{t}^{\prime} \Pi_{t}+C_{t}^{2}=0
$$

с граничным условием $\Pi_{T}^{T}=F_{T}^{2}$, и пусть $r^{T}=\left(r_{t}^{T}\right)_{t \leqslant T}$ - решение уравнения

$$
\dot{r}_{t}+\left(A_{t}^{\prime}-\Pi_{t} B_{t} B_{t}^{\prime}\right) r_{t}+\Pi_{t} l_{t}+m_{t}=0
$$

с граничным условием $r_{T}^{T}=n_{T}$.

Определим процесс $\widehat{x}^{T}=\left(\widehat{x}_{t}^{T}\right)_{t \leqslant T}$ как решение стохастического уравнения

$$
d \widehat{x}_{t}^{T}=\left(A_{t}-B_{t} B_{t}^{\prime} \Pi_{t}^{T}\right) \widehat{x}_{t}^{T}+\left(l_{t}-B B_{t}^{\prime} r_{t}^{T}\right) d t+G_{t} d w_{t}, \quad \widehat{x}_{0}^{T}=\xi
$$

Тогда

$$
\widehat{u}_{t}^{T}:=-B_{t}^{\prime}\left(\Pi_{t}^{T} \widehat{x}_{t}^{T}+r_{t}^{T}\right)
$$

является оптимальным управлением, которому соответствует фазовый процесс $\widehat{x}^{T}$.

Форма синтеза, соответствуюшего управлению $\widehat{u}^{T}$ в (12), не зависит от $G$. При естественных предположениях функции $\Pi^{T}$ и $r^{T}$ сходятся при $T \rightarrow \infty$ к некоторым функциям $\Pi$ и $r$, определенным на всей полуоси и не зависящим от $F$ и $n$. Эти функции определяют оптимальный синтез в задаче минимизации математического ожидания за единицу времени на бесконечном интервале. Как мы увидим позже, в однородном случае предполюжения теоремы 1 гарантируют существование соответствующих пределов. В общем случае мы приводим условия, которые гарантируют как существование пределов $\Pi_{t}=\lim _{T \rightarrow \infty} \Pi_{t}^{T}$ и $r_{t}=\lim _{T \rightarrow \infty} r_{t}^{T}$, так и выполнение приводимых ниже предположений.

А. Функции $A, B$ и $C$ таковы, что существует абсолютно непрерывная функция $\Pi=\left(\Pi_{t}\right)$, которая удовлетворяет уравнению Риккати (9) и такова, что фундаментальная матрица $\Psi(t, s)$ для функции

$$
\mathscr{A}_{t}:=A_{t}-B_{t} B_{t}^{\prime} \Pi_{t}
$$


допускает экспоненциальную оценку

$$
\|\Psi(t, s)\| \leqslant \gamma e^{-\kappa(t-s)}, \quad s \leqslant t
$$

с положительными константами $\gamma$ и $\kappa$.

Напомним, что $\Psi(t, s)$ является решением следующего матричного уравнения:

$$
\frac{\partial \Psi(t, s)}{\partial t}=\mathscr{A}_{t} \Psi(t, s), \quad \Psi(s, s)=I
$$

где $I$ - тождественная матрица. При этом $\Psi(t, s)=\Psi(t, 0) \Psi^{-1}(s, 0)$ и

$$
\frac{\partial \Psi(t, s)}{\partial s}=-\Psi(t, s) \mathscr{A}_{s}, \quad \Psi(s, s)=I .
$$

Следующая гипотеза описывает свойства детерминированного линейного регулятора

$$
d y_{t}=\left(A_{t} y_{t}+B_{t} v_{t}\right) d t, \quad y_{0}=0
$$

В. Существует такая константа $c_{0}>0$, что для любого $T>0$ и любой пары $\left(y_{t}, v_{t}\right)_{t \leqslant T}$, удовлетворяюшей $(16)$,

$$
\left\|y_{T}\right\|^{2}+\int_{0}^{T}\left\|y_{t}\right\|^{2} d t \leqslant c_{0} \int_{0}^{T}\left(\left\|C_{t} y_{t}\right\|^{2}+\left\|v_{t}\right\|^{2}\right) d t
$$

Положим

$$
\widehat{u}_{t}:=-B_{t}^{\prime}\left(\Pi_{t} \widehat{x}_{t}+r_{t}\right)
$$

где функция

$$
r_{t}=\int_{t}^{\infty} \Psi^{\prime}(v, t)\left(\Pi_{v} l_{v}+m_{v}\right) d v
$$

является решением уравнения (10), а $\widehat{x}$ - решением стохастического уравнения

$$
d \widehat{x}_{t}=\left(A_{t}-B_{t} B_{t}^{\prime} \Pi_{t}\right) \widehat{x}_{t} d t+\left(l_{t}-B_{t} B_{t}^{\prime} r_{t}\right) d t+G_{t} d w_{t}, \quad \widehat{x}_{0}=\xi .
$$

Очевидно, что $\widehat{x}$ - фазовый процесс, соответствующий управлению $\widehat{u}$.

Теорема 2. Пусть выполнены предположения А $и$ В. Тогда существует такая константа $b>0$, что функция $b \ln t$ является верхней функиией для всех проиессов дефекта.

3 а м е ч а н и е. Предположим, что $\Gamma_{t}$ - периодическая ограниченная функция. Рассмотрим модель (4), (7) с $F_{T}(x, u)=0$ и $f_{t}\left(x_{t}, u_{t}\right)=$ $\left\|C\left(x_{t}-\Gamma_{t}\right)\right\|^{2}+\left\|u_{t}\right\|^{2}$. Получается так называемая задача «трекинга» (см. [14]). Для этой модели справедливо утверждение теоремы, поскольку она является частным случаем (6) и (7) с $l_{t}=n_{t}=0$ и $m_{t}=\Gamma_{t}$. 


\section{3. Доказательство основного результата}

Пусть $z=(x, u)$ удовлетворяет $(6)$ на $[0, T]$, и пусть $\widehat{z}=(\widehat{x}, \widehat{u})$ задается соотношениями $(20),(18)$, где $\Pi_{t}$ и $r_{t}$ удовлетворяют $(9),(10)$ с произвольным граничным условием. Положим $y:=x-\widehat{x}, v:=u-\widehat{u}$. Тогда пара $(y, v)$ удовлетворяет $(16)$ и

$$
\begin{aligned}
\Delta_{T}:= & J_{T}(\widehat{z})-J_{T}(z)=-\left\|F_{T} y_{T}\right\|^{2}-2 y_{T}^{\prime}\left(F_{T} \widehat{x}_{T}+n_{T}\right) \\
& -\int_{0}^{T}\left(\left\|C_{t} y_{t}\right\|^{2}+\left\|v_{t}\right\|^{2}\right) d t-2 \int_{0}^{T}\left(y_{t}^{\prime} C_{t}^{2} \widehat{x}_{t}+v_{t}^{\prime} \widehat{u}_{t}-2 y_{t}^{\prime} m_{t}\right) d t .
\end{aligned}
$$

Используя (9), (10) и (20), нетрудно проверить, что

$$
d\left(\Pi_{t} \widehat{x}_{t}+r_{t}\right)=-\left(A_{t}^{\prime} \Pi_{t}+C_{t}^{2}\right) \widehat{x}_{t} d t-\left(A_{t}^{\prime} r_{t}+m_{t}\right) d t+\Pi_{t} G_{t} d w_{t} .
$$

Используя (16), (22) и (18), получаем:

$$
\begin{aligned}
d\left(y_{t}^{\prime}\left(\Pi_{t} \widehat{x}_{t}+r_{t}\right)\right) & =\left(y_{t}^{\prime} A_{t}^{\prime}+v_{t}^{\prime} B_{t}^{\prime}\right)\left(\Pi_{t} \widehat{x}_{t}+r_{t}\right) d t+y_{t}^{\prime} d\left(\Pi_{t} \widehat{x}_{t}+r_{t}\right) \\
& =-\left(y_{t}^{\prime} C_{t}^{2} \widehat{x}_{t}+v_{t}^{\prime} \widehat{u}_{t}+y_{t}^{\prime} m_{t}\right) d t+y_{t}^{\prime} \Pi_{t} G_{t} d w_{t} .
\end{aligned}
$$

Подставляя во второй интеграл в (21) выражение, получающееся из этого тождества, приходим к формуле:

$$
\begin{aligned}
\Delta_{T}= & -\left\|F_{T} y_{T}\right\|^{2}-\int_{0}^{T}\left(\left\|C_{t} y_{t}\right\|^{2}+\left\|v_{t}\right\|^{2}\right) d t \\
& +2 y_{T}^{\prime}\left(\left(\Pi_{T}-F_{T}^{2}\right) \widehat{x}_{T}+\left(r_{T}-n_{T}\right)\right)-2 \int_{0}^{T} y_{t}^{\prime} \Pi_{t} G_{t} d w_{t} .
\end{aligned}
$$

Если $\Pi_{t}=\Pi_{t}^{T}, r_{t}=r_{t}^{T}$, т.е. выполняются граничные условия $\Pi_{T}^{T}=F_{T}^{2}$ и $r_{T}^{T}=n_{T}$, то математическое ожидание левой части (23) неположительно, и, следовательно, $\widehat{x}_{t}^{T}$ является (единственным) оптимальным процессом в задаче (8).

Возьмем в (23) функцию $\Pi_{t}$ из $\mathbf{A}, r_{t}$ из (19). Элементарное неравенство

$$
2 a\left(d_{1}+d_{2}\right) \leqslant 2 c_{0}\left(d_{1}^{2}+d_{2}^{2}\right)+a^{2} c_{0}^{-1}
$$

и условие $\mathbf{B}$ приводят к неравенству

$\Delta_{T} \leqslant 2 c_{0}\left(\left\|\left(\Pi_{T}^{1 / 2}-F_{T}\right) \widehat{x}_{T}\right\|^{2}+\left(r_{T}-n_{T}\right)^{2}\right)-\frac{1}{c_{0}} \int_{0}^{T}\left\|y_{t}\right\|^{2} d t-2 \int_{0}^{T} y_{t}^{\prime} \Pi_{t} G_{t} d w_{t}$.

Из (19) и $\mathbf{A}$ следует, что функция $r_{T}$ ограничена. Поскольку $G, \Pi, F$ и $n$ также ограничены, то отсюда следует, что для некоторых $c_{1}, c_{2}>0$ выполняется:

$$
\Delta_{T} \leqslant c_{1}\left\|\widehat{x}_{T}\right\|^{2}+R_{T}
$$


где

$$
R_{T}=c_{2}-c_{1} \int_{0}^{T}\left\|G_{t}^{\prime} \Pi_{t} y_{t}\right\|^{2} d t-2 \int_{0}^{T} y_{t}^{\prime} \Pi_{t} G_{t} d w_{t}
$$

Утверждение теоремы следует из приведенной оценки. В самом деле, в соответствии с леммой А.1 из дополнения $\lim \sup _{T \rightarrow \infty} R_{T} g_{T} \leqslant 0$ даже для $g_{T}=o(1)$ при $T \rightarrow \infty$. Для оценки $\left\|\widehat{x}_{T}\right\|^{2}$ используем лемму А.2 из дополнения. Согласно (20) разность между $\widehat{x}_{T}$ и процессом, задаваемым (38) с $\mathscr{A}_{t}=A_{t}-B_{t} B_{t}^{\prime} \Pi_{t}$, равна

$$
\Psi(T, 0) \xi+\int_{0}^{T} \Psi(T, t)\left(l_{t}-B_{t} B_{t}^{\prime} r_{t}\right) d t
$$

Полученное выражение ограничено в силу ограниченности $l, r, B$ и экспоненциального неравенства (13). А поэтому, в силу леммы А.2,

$$
\limsup _{T \rightarrow \infty} \frac{\left\|\widehat{x}_{T}\right\|^{2}}{\ln T}<c
$$

Теорема доказана.

\section{4. О предположениях}

В этом разделе приводятся достаточные условия для выполнения предположений основной теоремы, формулируемые в стандартных терминах для модели, задаваемой дифференциальным уравнением (16), и доказывается теорема 1.

Обозначим $\Phi(t, s)$ фундаментальную матрицу, соответствующую матрице $A=\left(A_{t}\right)$. Поскольку $A$ ограничена, из леммы ГронуоллаБеллмана получаем экспоненциальную оценку

$$
\|\Phi(t, s)\| \leqslant e^{a(t-s)}, \quad t \geqslant s
$$

c $a:=\sup _{r}\left\|A_{r}\right\|$.

Положим

$$
\begin{aligned}
& W\left(t, t_{0}\right):=\int_{t_{0}}^{t} \Phi(t, s) B_{s} B_{s}^{\prime} \Phi^{\prime}(t, s) d s \\
& M\left(t, t_{0}\right):=\int_{t_{0}}^{t} \Phi^{\prime}\left(s, t_{0}\right) C_{s}^{2} \Phi\left(s, t_{0}\right) d s .
\end{aligned}
$$

Пара ограниченных матриц-функций $(A, B)$ называется равномерно вполне управляемой, если существуют такие положительные константы $\alpha$ и $\sigma$, что

$$
W(t+\sigma, t) \geqslant \alpha I \quad \text { для любого } t \geqslant 0,
$$


где знак неравенства между двумя матрицами означает, что их разность неотрицательно определена.

Пара ограниченных матриц-функций $(A, C)$ называется равномерно вполне восстанавливаемой, если существуют такие положительные константы $\beta$ и $h$, что

$$
M(t+h, t) \geqslant \beta I \quad \text { для любого } t \geqslant 0,
$$

или, что эквивалентно,

$$
\int_{t}^{t+h}\left\|C_{s} \Phi(s, t) \eta\right\|^{2} d s \geqslant \beta\|\eta\|^{2}
$$

для любых $t \geqslant 0$ и $\eta \in \mathbf{R}^{n}$.

Для объяснения смысла введенных понятий рассмотрим явное решение уравнения (16), задаваемое формулой Коши

$$
y_{t}=\Phi\left(t, t_{0}\right) y_{t_{0}}+\int_{t_{0}}^{t} \Phi(t, s) B_{s} v_{s} d s .
$$

В частности, для $v_{s}=B_{s}^{\prime} \Phi^{\prime}\left(t_{1}, s\right) \eta, s \in\left[t_{0}, t_{1}\right]$, получаем

$$
y_{t_{1}}=\Phi\left(t_{1}, t_{0}\right) y_{t_{0}}+W\left(t_{1}, t_{0}\right) \eta
$$

Таким образом, если матрица $W\left(t_{1}, t_{0}\right)$ обратима, то из любого состояния в момент $t_{0}$ можно попасть в любое состояние в момент $t_{1}$, т.е. система «управляема». Например, для того, чтобы попасть в момент $t_{1}$ в начало координат, находясь в момент $t_{0}$ в состоянии $y_{t_{0}}$, нужно на отрезке $\left[t_{0}, t_{1}\right]$ использовать управление, задаваемое формулой

$$
\bar{v}_{s}=-B_{s}^{\prime} \Phi^{\prime}\left(t_{1}, s\right) W^{-1}\left(t_{1}, t_{0}\right) \Phi\left(t_{1}, t_{0}\right) y_{t_{0}} .
$$

Матрица $W^{-1}\left(t_{1}, t_{0}\right)$ связана с минимальной «энергией управления» (измеряемой как интеграл от $\left\|v_{s}\right\|^{2}$ ), необходимой для перехода из одного состояния в другое. Равномерная управляемость гарантирует не только возможность перехода, но и то свойство, что энергия управления и время перехода не зависят от начального и конечного состояний.

Равномерная восстанавливаемость гарантирует, что, зная значение $y_{t+h}$ и значения $z_{s}=C_{s} y_{s}$ при всех $s \in[t, t+h]$, можно определить значение $y_{t}$.

3 а м е ч а н и е. Если $A$ и $C$ ограничены и $\left|\operatorname{det} C_{t}\right| \geqslant \varepsilon$ для некоторого $\varepsilon>0$, то пара $(A, C)$ равномерно вполне восстанавливаема.

Лемма 1. Любое из следуюших двух условий обеспечивает выполнение условия $\mathbf{B}$ :

(i) пара $(A, C)$ равномерно вполне восстанавливаема; 
(ii) Функиия $A$ такова, что соответствующая ей фундаментальная матрица $\Phi(t, s)$ допускает экспоненииальную оченку

$$
\|\Phi(t, s)\| \leqslant \gamma_{1} e^{-\kappa_{1}(t-s)}, \quad t \geqslant s
$$

для некоторых $\gamma_{1}>0$ u $\kappa_{1}>0$.

Д ок а з а т ел в с тво. Рассмотрим случай (i). Положим $v(t, s):=\int_{s}^{t} \Phi(t, r) B_{r} v_{r} d r, s \leqslant t$. Используя неравенство Буняковского, экспоненциальное неравенство (26), и ограниченность $B$, легко получить, что для любого $t \leqslant s+h$

$$
\|v(t, s)\|^{2} \leqslant \int_{s}^{t}\left\|\Phi(t, r) B_{r}\right\|^{2} d r \int_{s}^{t}\left\|v_{r}\right\|^{2} d r \leqslant c \int_{s}^{s+h}\left\|v_{r}\right\|^{2} d r .
$$

Здесь и в дальнейшем $c$ обозначает константу, точное значение которой не играет роли и которая меняется от формулы к формуле.

Из представления $\Phi(\theta, t) y_{t}=y_{\theta}-v(\theta, t)$ (которое представляет собой просто формулу Коши) и из (33) вытекает, что

$$
\left\|C_{\theta} \Phi(\theta, t) y_{t}\right\|^{2} \leqslant 2\left(\left\|C_{\theta} y_{\theta}\right\|^{2}+\left\|C_{\theta} v(\theta, t)\right\|^{2}\right) \leqslant c\left(\left\|C_{\theta} y_{\theta}\right\|^{2}+\int_{t}^{t+h}\left\|v_{r}\right\|^{2} d r\right)
$$

при $t \leqslant \theta \leqslant t+h \leqslant T$. Подставляя $y_{t}$ вместо $\eta$ в (29) и используя полученную оценку, получаем, что для $t \leqslant T-h$

$$
\beta\left\|y_{t}\right\|^{2} \leqslant \int_{t}^{t+h}\left\|C_{\theta} \Phi(\theta, t) y_{t}\right\|^{2} d \theta \leqslant c \int_{t}^{t+h}\left(\left\|C_{s} y_{s}\right\|^{2}+\left\|v_{s}\right\|^{2}\right) d s .
$$

Интегрируя полученное неравенство на $[0, T-h]$ и изменяя порядок интегрирования, приходим к оценке

$$
\int_{0}^{T-h}\left\|y_{t}\right\|^{2} d t \leqslant c \int_{0}^{T}\left(\left\|C_{t} y_{t}\right\|^{2}+\left\|v_{t}\right\|^{2}\right) d t .
$$

Для $t \in[T-h, T]$, используя аналогичные выкладки, получаем с помощью (34), что

$$
\left\|y_{t}\right\|^{2} \leqslant 2\left(\left\|\Phi(t, T-h) y_{T-h}\right\|^{2}+\|v(t, T-h)\|^{2} \leqslant c \int_{0}^{T}\left(\left\|C_{t} y_{t}\right\|^{2}+\left\|v_{t}\right\|^{2}\right) d t .\right.
$$

Таким образом, интеграл от $\left\|y_{t}\right\|^{2}$ по $[T-h, T]$ также оценивается левой частью (35) с точностью до изменения константы. То же самое справедливо для $\left\|y_{T}\right\|^{2}$, а значит, В выполняется.

В случае (ii) можно получить следуюшую оценку, которая лучше, чем (17):

$$
\left\|y_{T}\right\|^{2}+\int_{0}^{T}\left\|y_{t}\right\|^{2} d t \leqslant c_{0} \int_{0}^{T}\left\|v_{t}\right\|^{2} d t
$$


В самом деле, используя формулу Коши (30), ограниченность $B$ и (32), получаем

$$
\left\|y_{t}\right\|=\left\|\int_{0}^{t} \Phi(t, s) B_{s} v_{s} d s\right\| \leqslant c \int_{0}^{t} e^{-\kappa_{1}(t-s)}\left\|v_{s}\right\| d s .
$$

Таким образом, в силу неравенства Буняковского

$$
\left\|y_{t}\right\|^{2} \leqslant \frac{c^{2}}{\kappa_{1}} \int_{0}^{t} e^{-\kappa_{1}(t-s)}\left\|v_{s}\right\|^{2} d s
$$

I

$$
\int_{0}^{T}\left\|y_{t}\right\|^{2} d t \leqslant \frac{c^{2}}{\kappa_{1}} \int_{0}^{T} \int_{0}^{t} e^{-\kappa_{1}(t-s)}\left\|v_{s}\right\|^{2} d s d t \leqslant \frac{c^{2}}{\kappa_{1}^{2}} \int_{0}^{T}\left\|v_{s}\right\|^{2} d s,
$$

что завершает доказательство результата.

Предложение 1. Любое из следующих двух условий обеспечивает выполнение $\mathbf{A}, \mathbf{B}$ и сходимость $\Pi^{T} \kappa \Pi$ и $r^{T} \kappa r$ :

(i) пара $(A, B)$ равномерно вполне управляема, а пара $(A, C)$ равномерно вполне восстанавливаема;

(ii) Функиия $A$ такова, что соответствуюшая ей фундаментальная матрица $\Phi(t, s)$ допускает экспоненциальную оченку (32).

Д ок а з а т льс т в о. При наших предположениях сходимость $\Pi^{T}$ к решению уравнения Риккати и справедливость экспоненциальной оценки (13) для $\Psi^{T}$ и $\Psi$ хорошо известны (см., например, [13]). В соответствии с (10)

$$
r_{t}^{T}=\Psi^{T \prime}(T, t) n_{T}+\int_{t}^{T} \Psi^{T^{\prime}}(u, t)\left(\Pi_{u}^{T} l_{u}+m_{u}\right) d u
$$

и сходимость $r^{T}$ к $r$, которое определяется из (19), следует из сходимости $\Pi^{T}$ и экспоненциальной оценки $(13)$ для $\Psi^{T}$. Таким образом, остается доказать только условие А. Хорошо известно (см., например, [13]), что для детерминированной оптимизационной задачи на $\left[t_{0}, \infty[\right.$ с $l=m=$ $n=0, F_{T}=0$ и с начальным состоянием $y_{t_{0}}$ минимальное значение функционала равно $\left\|\Pi_{t_{0}}^{1 / 2} y_{t_{0}}\right\|^{2}$. Таким образом, остается проверить, что последнее свойство П вместе с предположением о том, что $(A, B)$ равномерно вполне управляемы, обеспечивает ограниченность П. Для доказательства этого факта в случае (i) рассмотрим на $\left[t_{0}, \infty[\right.$ пару $(\bar{y}, \bar{v})$, где управление $\bar{v}$ задается формулой $(31)$ на интервале $\left[t_{0}, t_{1}\right], t_{1}:=t_{0}+\sigma$, и равно нулю вне этого интервала. Автоматически, $\bar{y}_{t}=0$ для всех $t \geqslant t_{1}$. В силу определения оптимальности

$$
\left\|\Pi_{t_{0}}^{1 / 2} y_{t_{0}}\right\|^{2} \leqslant \int_{t_{0}}^{t_{1}}\left(\left\|C_{s} \bar{y}_{s}\right\|^{2}+\left\|\bar{v}_{s}\right\|^{2}\right) d s .
$$

Из определения $\bar{v}$ и $W\left(t_{1}, t_{0}\right)$ вытекает, что

$$
\int_{t_{0}}^{t_{1}}\left\|\bar{v}_{s}\right\|^{2} d s=y_{t_{0}} \Phi^{\prime}\left(t_{1}, t_{0}\right) W^{-1}\left(t_{1}, t_{0}\right) \Phi\left(t_{1}, t_{0}\right) y_{t_{0}} \leqslant c\left\|y_{t_{0}}\right\|^{2} .
$$


Формула Коши (30) позволяет получить аналогичную оценку для интеграла от $\left\|C_{s} \bar{y}_{s}\right\|^{2}$. Таким образом, $\left\|\Pi_{t_{0}}^{1 / 2} y_{t_{0}}\right\| \leqslant c\left\|y_{t_{0}}\right\|$, что доказывает результат для случая (i).

В случае (ii) рассмотрим на $\left[t_{0}, \infty[\right.$ пару $(\bar{y}, \bar{v})$, где $\bar{v}$ тождественно равно нулю. В силу свойства оптимальности, ограниченности $C$ и экспоненциальной оценки для $\bar{y}$ получаем

$$
\left\|\Pi_{t_{0}}^{1 / 2} y_{t_{0}}\right\|^{2} \leqslant \int_{t_{0}}^{\infty}\left\|C_{s} \bar{y}_{s}\right\|^{2} d s \leqslant c\left\|\bar{y}_{t_{0}}\right\|^{2} .
$$

Это завершает доказательство теоремы.

Приведем теперь достаточные условия, гарантирующие выполнение предположений теоремы 2 в случае постоянных коэффициентов.

Напомним, что пара матриц $(A, B)$ называется управляемой, если матрица $\left[B: A B: \cdots: A^{n-1} B\right]$ имеет полный ранг. Пара матриц $(A, C)$ является наблюдаемой, если $\left(A^{\prime}, C^{\prime}\right)$ управляема.

Легко проверить, что пара постоянных матриц-функций $A=A_{t}$, $B=B_{t}$ равномерно вполне управляема тогда и только тогда, когда пара $(A, B)$ управляема; аналогичное соответствие имеет место между понятиями равномерной вполне восстанавливаемости и наблюдаемости (см. [13]). Поэтому из предложения 1 непосредственно следует:

Предложение 2. Предположим, что в модели с постоянными коэффиииентами пара $(A, B)$ управляема, а пара $(A, C)$ наблюдаема. Тогда П не зависит от $t$ и выполняются условия $\mathbf{A} u \mathbf{B}$.

Оказывается, что приведенные условия можно ослабить. А именно, справедливо следующее утверждение, которое превращает теорему 1 в следствие теоремы 2.

Предложение 3. Предположим, что в модели с постоянньми коэффиииентами пара $(A, B)$ стабилизируема, а пара $(A, C)$ выляляема. Тогда П не зависит от $t$ и выполняются условия $\mathbf{A} u \mathbf{B}$.

Используемые в формулировке понятия имеют следующий смысл. Пара матриц $(A, B)$ называется стабилизируемой, если сушествует такая матрица $K$, что матрица $A+B K$ устойчива. Пара матриц $(A, C)$ является вылявляемой, если $\left(A^{\prime}, C^{\prime}\right)$ стабилизируема. Из управляемости следует стабилизируемость, а значит, из наблюдаемости следует выявляемость (см., например, [8]).

Хорошо известно (см. [13])), что условие $\mathbf{A}$ остается верным при ослабленных предположениях, поэтому остается доказать В. Оказывается, что это утверждение предположения 3 , будучи формально более общим, на самом деле является простым следствием соответствующего утверждения предложения 2 в силу теоремы разложения, которая позволяет представить в соответствующем базисе модель с выявляемыми $(A, C)$ в так называемой канонической форме наблюдаемости 
(см. [13]). Если с самого начала модель записана в этом базисе, то

$$
A=\left(\begin{array}{cc}
A_{11} & 0 \\
A_{21} & A_{22}
\end{array}\right), \quad C=\left(\begin{array}{cc}
C_{11} & 0 \\
0 & 0
\end{array}\right)
$$

где пара $\left(A_{11}, C_{11}\right)$ наблюдаема, а матрица $A_{22}$ устойчива. Неравенство (17) можно переписать в виде

$$
\left\|y_{T}\right\|^{2}+\int_{0}^{T}\left\|y_{t}\right\|^{2} d t \leqslant c_{0} \int_{0}^{T}\left(\left\|C_{11} y_{1 t}\right\|^{2}+\left\|v_{t}\right\|^{2}\right) d t
$$

где $y_{1 t}$ (наблюдаемая часть $y_{t}$ ) включает только координаты $y_{t}$, соответствующие матрице $A_{11}$. Ясно, что соответствующее утверждение можно доказать, рассматривая отдельно траекторию $y_{1 t}$ (для которой результат уже известен) и $y_{t 2}$, которая включает остальные координаты. Поскольку матрица $A_{22}$ устойчива, можно воспользоваться леммой 1 (ii) с «управлением» $A_{21} y_{1 t}+B_{2} v_{t}$.

\section{Дополнение: два вероятностных утверждения}

Для удобства читателей мы приводим версию усиленного закона больших чисел для непрерывного локального мартингала и утверждение о скорости роста «обобщенного» процесса Орнштейна-Уленбека, которое является простым следствием закона повторного логарифма для винеровского процесса.

Лемма А.1. Пусть $M-$ локальньий мартингал, $M_{0}=0, u c>0-$ некоторая константа. Тогда

(a) $M_{T}-c\langle M\rangle_{T} \rightarrow-\infty$ n.н. на $\left\{\langle M\rangle_{\infty}=\infty\right\}$,

(b) $M_{T}-c\langle M\rangle_{T} \rightarrow M_{\infty}-c\langle M\rangle_{\infty}$ n.н. на $\left\{\langle M\rangle_{\infty}<\infty\right\}$.

Д о к а з а т е л ь с т в о. Это утверждение следует из двух хорошо известных фактов (см., например, [22]):

$$
\begin{gathered}
\left\{\langle M\rangle_{\infty}<\infty\right\}=\left\{\lim _{t \rightarrow \infty} M_{t} \text { сушествует и конечен }\right\} \text { п.н., } \\
\lim _{t \rightarrow \infty} M_{t} /\langle M\rangle_{t}=0 \quad \text { п.н. на } \quad\left\{\langle M\rangle_{\infty}=\infty\right\} .
\end{gathered}
$$

Лемма А.2. Пусть $x$ - решение линейного стохастического уравнения

$$
d x_{t}=\mathscr{A}_{t} x_{t} d t+G_{t} d w_{t}, \quad x_{0}=0
$$

где $\mathscr{A}$ и - ограниченнье предсказуемые прочессы, а фундаментальная матрица $\Psi(t, s)$ допускает экспоненииальную оченку

$$
\|\Psi(t, s)\| \leqslant \gamma e^{-\kappa(t-s)}, \quad s \leqslant t .
$$

Tozda

$$
\limsup _{T \rightarrow \infty} \frac{\left\|x_{T}\right\|}{\sqrt{\ln T}}<\text { const. }
$$


Д о к а з а т е л ь с т в о. Сначала мы докажем (40) для процесса $\widetilde{x}$, который является решением (38) с $\mathscr{A}=-I$. Легко видеть, что в этом случае достаточно рассмотреть скалярную задачу

$$
d \widetilde{x}_{t}=-\widetilde{x}_{t} d t+d m_{t}, \quad \widetilde{x}_{0}=0,
$$

где $m$ - непрерывный мартингал с $d\langle m\rangle / d t \leqslant 1$.

Решая приведенное выше уравнение и используя классическую замену времени (которая требует расширения вероятностного пространства), получаем, что

$$
\widetilde{x}_{t}=e^{-t} \int_{0}^{t} e^{s} d m_{s}=e^{-t} M_{t}=e^{-t} W_{\langle M\rangle_{t}},
$$

где $W$ - винеровский процесс и $\langle M\rangle_{t} \leqslant e^{2 t}$. Таким образом,

$$
\frac{\left|\widetilde{x}_{T}\right|}{\sqrt{\ln 2 T}}=\frac{\left|W_{\langle M\rangle_{T}}\right|}{e^{T} \sqrt{\ln 2 T}} \leqslant \frac{\left|W_{\langle M\rangle_{T}}\right|}{\sqrt{\langle M\rangle_{t} \ln \ln \langle M\rangle_{T}}} .
$$

Представление (41) показывает, что на множестве $\left\{\langle M\rangle_{\infty}<\infty\right\}$ предел $\widetilde{x}_{t}$ равен нулю. На дополнении этого множества можно применить закон повторного логарифма для винеровского процесса.

Таким образом, (40) выполняется в случае, когда $\mathscr{A}=-I$. Для того чтобы получить результат для обшего случая, положим $\Delta:=x-\widetilde{x}$. Тогда $d \Delta_{t}=\mathscr{A}_{t} \Delta_{t} d t+\left(\mathscr{A}_{t}+I\right) \widetilde{x}_{t} d t, \Delta_{0}=0$. Следовательно,

$$
\Delta_{t}=\int_{0}^{t} \Psi(t, s)\left(\mathscr{A}_{s}+I\right) \tilde{x}_{s} d s .
$$

Выше мы доказали, что существует такая константа $c$, что для некоторого $t_{0}=t_{0}(\omega)$ справедливо $\sup _{s} \geqslant t_{0}\left\|\widetilde{x}_{s}\right\| / \sqrt{\ln s} \leqslant c$. Из ограниченности $\mathscr{A}$ и экспоненциальной оценки (39) вытекает, что

$$
\frac{\left\|\Delta_{T}\right\|}{\sqrt{\ln T}} \leqslant \frac{1}{\sqrt{\ln T}} \int_{0}^{t_{0}} \Psi\left(t_{0}, s\right)\left(\mathscr{A}_{s}+I\right) \widetilde{x}_{s} d s+C \sup _{t_{0} \leqslant t \leqslant T} \frac{\left\|\widetilde{x}_{t}\right\|}{\sqrt{\ln t}}
$$

для некоторой константы $C$. Тем самым утверждение доказано. ${ }^{1)}$

\section{СПИСОК ЛИТЕРАТУРЫ}

1. Asriev A. V., Rotar' V.I. On asymptotic optimality in probability and almost surely in dynamic control. - Stochastics Stochastics Rep., 1990, v. 33, p. 1-16.

2. Белкина Т.А., Пресман Э. Л. Асимптотически оптимальные по распределению управления для линейной стохастической системы с квацратичным функционалом. - Автоматика и телемеханика, 1997, т. 58, в. 3, с. 106-115.

1) Первоначальная версия этой статьи была представлена авторами для публикации в SIAM Journal on Control and Optimization (SICON) и была отклонена этим журналом на основании отзыва, который, по убеждению авторов, содержит ошибочные утверждения. Подробности переписки авторов с редакцией SICON можно найти на сайте www.gcnet.ru/ elpresma. - Прим. автора. 
3. Белкина Т. А., Ротарь В. И. Об условиях асимптотической оптимальности по вероятности и почти наверное в модели управляемого диффузионного процесса. Автоматика и телемеханика, 1999, т. 60, в. 2, с. 46-56.

4. Bensoussan A. Stochastic Control of Partially Observable Systems. Cambridge: Cambridge University Press, 1992.

5. Borkar V.S. Optimal Control of Diffusion Processes (Pitman Research Notes in Mathematics Series, 203). Harlow: Longman, 1989.

6. Carlson D. A., Haurie A. B., Leizarowitz A. Infinite Horizon Optimal Control. Deterministic and Stochastic Systems. Berlin: Springer-Verlag, 1991.

7. Dai Pra P., Di Masi G. B., Trivellato B. Almost sure optimality and optimality in probability for stochastic control problems over an infinite time horizon. - Ann. Oper. Res., 1999, v. 88, p. 161-171.

8. Дэвис М.X.А. Линейное оценивание и стохастическое управление. М.: Наука, 1984, $202 \mathrm{c}$.

9. Di Masi G. B., Kabanov Yu. On sensitive probabilistic criteria in the linear regulator problem with the infinite horizon. - Обозрение прикладной и промышленной математики, 1998 , т. 5 , в. 2 , с. $410-422$.

10. Föllmer H., Majumdar M. On the asymptotic behavior of stochastic economic processes. Two examples from intertemporal allocation under uncertainty. - J. Math. Econom., 1978, v. 5, p. 275-287.

11. Конюхова (Белкина) T. А. Асимптотически оптимальные по вероятности управления в задаче о линейном регуляторе с переменными параметрами. - Автоматика и телемеханика, 1994, т. 55 , в. 2 , с. 110-120.

12. Конюхова (Белкина) Т.А., Ротарь В.И. Управления, асимптотически оптимальные по вероятнссти и почти наверное в задаче о линейном регуляторе. Автоматика и телемеханика, 1992, т. 53, в. 6, с. 65-78.

13. Kwakernaak H., Sivan R. Linear Optimal Control Systems. New York-London: Wiley-Interscience, 1972.

14. Leizarowitz $A$. Infinite horizon stochastic regulation and tracking with the overtaking criteria. - Stochastics, 1987, v. 22, p. 117-150.

15. Leizarowitz $A$. On almost sure optimization for stochastic controls systems. Stochastics, 1988 , v. 23, p. 85-107.

16. Lippman S. Maximal average-reward policies for semi-Markov decision processes with arbitrary state and action space. - Ann. Math. Statist., 1971, v. 42, p. 1717-1726.

17. Mandl $P$. Asymptotic ordering of probability distributions for linear controlled systems with quadratic cost. - Stochastic Differential Systems. Eds. N. Christopeit, K. Helmes, M. Kohlmann. Lecture Notes in Control and Inform. Sci., Berlin-New York: Springer-Verlag, 1986, p. 277-283.

18. Mandl P., Romera Ayllón M. R. On controlled Markov processes with average cost criterion. - Kybernetika (Prague), 1987, v. 23, p. 433-442.

19. Нагаев С. В., Пресман Э. Л. Закон повторного логарифма в одной задаче управления. - Теория вероятн. и ее примен., 1998, т. 43, в. 2, с. 364-369.

20. Пресман Э. Л. Оптимальность почти наверное и по вероятности для стохастического линейно-квадратического регулятора. - Теория вероятн. и ее примен., 1997, т. 42 , в. 3 , с. $627-632$.

21. Presman E., Rotar'V., Taksar M. Optimality in probability and almost surely. The general scheme and a linear regulator problem. - Stochastics Stochastics Rep., 1993, v. 43, p. $127-137$.

22. Revuz D., Yor M. Continuous Martingales and Brownian Motion. Berlin: SpringerVerlag, 1991.

23. Rotar' $V . I$. The recurrency property and optimality almost surely and in probability. New Trends in Probability and Statistics. 1. Proceedings of Bakuriany Colloquium in Honor of Yu. V. Prohorov. Utrecht: VSP, 1990. 